\title{
Netrin-1 Is Required for the Normal Development of Spinal Cord Oligodendrocytes
}

\author{
Hui-Hsin Tsai, ${ }^{1}$ Wendy B. Macklin, ${ }^{2}$ and Robert H. Miller ${ }^{1}$ \\ ${ }^{1}$ Department of Neurosciences, Case Western Reserve University, Cleveland, Ohio 44106, and ${ }^{2}$ Cleveland Clinic Foundation, Department of Neurosciences, \\ Lerner Research Center, Cleveland, Ohio 44195
}

\begin{abstract}
Successful CNS myelination is dependent on the correct localization of oligodendrocytes and their interactions with adjacent axons. In the spinal cord, oligodendrocyte precursors originate at the ventral midline and subsequently migrate to the white matter where they mature. In vitro studies suggest this dispersal is mediated by the guidance molecule netrin-1. Here, we show that in the spinal cord of netrin-1 mutant mice, oligodendrocyte precursors failed to disperse from the ventral midline as a consequence of a lack of polarization and directional migration. The lack of netrin-1 also resulted in an overall reduction of oligodendrocyte lineage cells that was independent of the failure of initial dispersal. Oligodendrocyte precursors injected into presumptive white matter underwent extensive radial migration and expansion in wild-type but not netrin-1 mutant hosts. These data indicate that netrin-1 is crucial for both the initial dispersal of spinal cord oligodendrocyte precursors and their subsequent development in the presumptive white matter.
\end{abstract}

Key words: oligodendrocyte precursor; migration; netrin-1; cytoarchitecture; time-lapse imaging; slice cultures

\section{Introduction}

Cell migration is a hallmark of development. In the vertebrate CNS, specific populations of neurons and glia generated at distinct locations migrate long distances to their final destinations (Hatten, 2002; Marin and Rubenstein, 2003; Jarjour and Kennedy, 2004).

Oligodendrocytes of the vertebrate CNS form myelin sheaths that wrap target axons to facilitate rapid axonal conduction (Bhat, 2003) and are predominantly located in white matter tracts. In the developing spinal cord, the majority of oligodendrocyte precursors (OPCs) arise in the ventral ventricular zone (Miller, 1996; Pringle et al., 1996) in a common domain with motor neurons (Rowitch, 2004). Their origin is specified by the opposing gradients of sonic hedgehog and bone morphogenetic protein 4 (Pringle et al., 1996; Mekki-Dauriac et al., 2002) that regulate expression of transcription factors establishing glial fate (Rowitch, 2004). OPCs migrate widely throughout the CNS and respond to various trophic factors (Barres and Raff, 1999; Miller, 2002) before differentiating and myelinating axons.

Although long-distance OPC migration has been well described (Small et al., 1987), the mechanisms underlying its guidance are less clear. One potential guidance molecule is netrin-1, a laminin-like molecule expressed at the midline that is important for axonal navigation and neuronal migration in the CNS

Received Aug. 23, 2005; revised Dec. 25, 2005; accepted Dec. 29, 2005.

This work was supported by National Institutes of Health Grants NS30800 and NS36674 (R.H.M.). We thank Dr. M. Qiu for PDGF $\alpha$ R and MBP plasmids, Dr. C. Stiles for Olig2 antibodies, and Dr. S. Robinson for critically reviewing this manuscript. We also thank R. Wang and V. DeGeorge for their technical assistance.

Correspondence should be addressed to Robert H. Miller, Department of Neurosciences, Case Western Reserve University, School of Medicine, 10900 Euclid Avenue, Cleveland, OH 44106. E-mail: rhm3@case.edu.

DOI:10.1523/JNEUROSCI.3571-05.2006

Copyright $\odot 2006$ Society for Neuroscience $\quad 0270-6474 / 06 / 261913-10 \$ 15.00 / 0$
(Kennedy et al., 1994; Serafini et al., 1994). Netrin can function as either a chemoattractant or a chemorepellent depending on the cellular context and receptor complexes. Chemoattraction of netrin-1 is mediated by receptors deleted in colorectal cancer (DCC) or neogenin (Keino-Masu et al., 1996), whereas chemorepulsion is mediated by expression of both DCC and Unc5 or Unc5 alone (Hong et al., 1999; Keleman and Dickson, 2001). The regulatory mechanisms responsible for neuronal guidance and migration (Guan and Rao, 2003) appear to be partially shared with glial cells. For example, netrin-1 has been proposed as a chemorepellent or dispersal factor for OPCs in vitro mediated by a combination of DCC and Unc5 receptors (Sugimoto et al., 2001; Jarjour et al., 2003; Tsai et al., 2003). In Drosophila, unc-5 is expressed in glia and a subset of neurons migrating away from the midline cells expressing netrin-1. Furthermore, embryos with panglial unc- 5 overexpression have increased numbers of laterally migrating glia (Freeman et al., 2003), suggesting that unc-5 mediates repulsive signal from netrin-1 in these cells.

To elucidate the role of netrin-1 in OPC migration in the murine spinal cord, we analyzed netrin-1 mutants and demonstrate an inhibition of OPC dispersal and a significant reduction in numbers of oligodendrocyte lineage cells in the absence of netrin-1. The dispersal defect reflects a lack of directional migration from the ventricular zone, whereas the loss of oligodendrocyte lineage cells reflects the role of netrin-1 in patterning the cytoarchitecture of white matter. Defective dispersal could not be recovered by direct transplantation of OPCs into presumptive white matter of netrin-1 mutants. These data suggest that netrin-1 is critical for both OPC dispersal from the ventral ventricular zone and for providing an intact developing white matter environment that supports the generation of the appropriate numbers of oligodendrocytes. 


\section{Materials and Methods}

Animals, antibodies, and statistics. Netrin-1 mutant mice were a gift from Dr. Tessier-Lavigne (Genentech, South San Francisco, CA) and were maintained in CD-1 background and in compliance with the animal protocol at Case Western Reserve University. The day the animals were plugged was designated as embryonic day 0 (E0). Timed animals were genotyped first using 5-bromo-4-chloro-3-indolyl- $\beta$-D-galactopyranoside (X-gal) to detect the mutant allele and further confirmed by reverse transcription (RT)-PCR genotyping (Serafini et al., 1996). The netrin-1/proteolipid protein (PLP)-enhanced green fluorescent protein (EGFP) animals were generated by crossing netrin-1 heterozygotes with PLP-EGFP transgenic mice in C57BL/6 and maintained as double heterozygotes. For mutant phenotype analysis, embryos were immersed in $4 \%$ PFA overnight and cut on a vibrating microtome (Leica VT1000S; Leica, Bannockburn, IL) at $50 \mu \mathrm{m}$. At least an independent set of three littermates for control and mutant animals were analyzed for each experiment, and animals were not shared with another analysis. Images were taken by confocal microscope (Zeiss, Thornwood, NY) at different magnifications indicated by scale bars in figure legends unless stated otherwise. Longitudinal sections from postnatal day 0 (P0) spinal cords were cut at $70 \mu \mathrm{m}$, and PLP-EGFP+ cells were counted from intact sections spanning from cervical to lumbar regions. Approximately five sections were counted for each animal, and cell numbers were expressed as the number of PLP-EGFP+ cells/section. All spinal cord coronal sections were compared at the same level of wild-type and mutant animals. Because netrin-1 mutant brains had defective corpus callosum, hippocampal, and anterior commissure (Serafini et al., 1996), special care was taken to ensure that the sections compared between wild-type and mutant animals were at the similar level close to the midline. Anti-GFAP antibodies were obtained from DAKO (1:200; DAKO, Carpinteria, CA). Anti-Olig2 antibodies were a gift from Dr. C. Stiles (Harvard University, Cambridge, MA) and used at 1:20,000. All data in this study comparing wild-type and mutant animals were analyzed by $t$ test, and the data are expressed as mean \pm SEM unless stated otherwise.

In situ hybridization. Timed embryos were collected and fixed with $4 \%$ paraformaldehyde (PFA) overnight at $4^{\circ} \mathrm{C}$, and $20 \mu \mathrm{m}$ sections of spinal cords were cut on a cryostat. Platelet-derived growth factor $\alpha$ receptor ( $\mathrm{PDGF} \alpha \mathrm{R})$ and myelin basic protein (MBP) in situ hybridization was done as described previously (Qi et al., 2001). Briefly, cryosections were pretreated and prehybridized in the hybridization solution for $2 \mathrm{~h}$ at room temperature. Hybridization solution containing $1 \mu \mathrm{g} / \mathrm{ml}$ RNA probes (Roche, Indianapolis, IN) were then added to the slides and incubated at $65^{\circ} \mathrm{C}$ overnight. After washing, sections were subsequently incubated with alkaline phosphatase-conjugated anti-digoxigenin antibody (1:5000; Roche) overnight and developed with nitrobluetetrazolium-chloride/5-bromo-4-chlor-indolyl-phosphate solution.

Time-lapse imaging. For analyzing migration of purified OPCs in the presence of netrin-1, P1 spinal cord A2B5+ cells from rat pups were immunopan-purified as described previously (Tsai et al., 2002) and plated on glass-bottom dishes overnight with or without mouse netrin-1 (R \& D Systems, Minneapolis, MN) at $100 \mathrm{ng} / \mathrm{ml}$. Fresh medium with 25 mM HEPES was added before recording the next day. Images were taken every $5 \mathrm{~min}$ for $3 \mathrm{~h}$ by a cooled CCD (Princeton Instruments, Trenton, $\mathrm{NJ})$ and inverted microscope (Axiovert 405M; Zeiss) under a $20 \times \mathrm{LD}$ (numerical aperture, 0.4 ) objective, and the migration velocity was calculated by MetaMorph (Fryer Company, Huntley, IL).

Explant cultures, cell proliferation, and cell death assays. Spinal cords from various embryonic ages of netrin-1 mice were dissected into dorsal and ventral regions. Ventral spinal cord explants were embedded in matrigel (growth factor-reduced; BD Biosciences, San Jose, CA) and cultured for 3 or $5 \mathrm{~d}$ before fixed by 4\% PFA and labeled by $\mathrm{O} 4$ antibodies.

For cell proliferation analyses, pregnant netrin-1 mice at E16.5 received multiple injections of $1 \%$ bromodeoxyuridine (BrdU) in normal saline during a $16 \mathrm{~h}$ period. The spinal cords were dissected and cut for the anti-BrdU staining to detect proliferating cells in vivo. To confirm the cell identity of the proliferating cells, pregnant netrin-1 mice at E16.5 received one injection of $1 \%$ BrdU 2 h before they were killed. Whole spinal cord cell cultures were established for individual embryos that were subsequently genotyped. After $15 \mathrm{~h}$ in culture, cells were labeled with anti-NG2 (Chemicon, Temecula, CA) and anti-BrdU (Roche) antibodies. The result was expressed as a percentage of $\mathrm{NG} 2+/ \mathrm{BrdU}+$ cells in the total $\mathrm{NG} 2+$ cell population. For cell death detection, E17 spinal cord sections were stained by activated caspase- 3 antibodies (Cell Signaling Technology, Beverly, MA) or the In Situ Cell Death Detection kit (Roche).

MTT assay for cell viability. A2B5+ and $\mathrm{O} 4+\mathrm{OPCs}$ were pan-purified and $\sim 4 \times 10^{4}$ cells plated in each well in a 96-well plate. Cells cultured in DMEM served as negative controls, and cells cultured in complete medium with growth factors served as positive controls. Mouse netrin-1 (R \& D Systems), anti-DCC function blocking antibodies (EMD Biosciences, La Jolla, CA), and control mouse IgG antibodies (R \& D Systems) were added as indicated. After $3 \mathrm{~d}$ in culture, MTT assay (Roche) was performed to measure cell viability and results read by the ELISA reader. Each condition was done in triplicates from three different preparations.

Slice injection. Slice cultures for cell injection $(500 \mu \mathrm{m})$ were prepared from E18 netrin-1 littermates. OPCs for injections were prepared in two ways. For the PLP-EGFP+ cells, P1-P4 spinal cords from PLP-EGFP transgenic animals were triturated and resuspended at a density of $4 \times$ $10^{4} \mathrm{cells} / \mu \mathrm{l}$. In each injection, $0.2 \mu \mathrm{l}$ of the cell suspension was used at an injection rate of $2 \mu \mathrm{l} / \mathrm{min}$. For A2B5+ cells, purified A2B5 OPCs from P1 rat spinal cords were labeled with the green cell tracker 5-chloromethylfluorescein diacetate (Invitrogen, Carlsbad, CA) at $10 \mu \mathrm{M}$ for $30 \mathrm{~min}$, rinsed with warm DMEM for $30 \mathrm{~min}$, and resuspended at a density of $4 \times 10^{4}$ cells $/ \mu$ l. After injection, the slice cultures were kept on polycarbonate inserts for $2 \mathrm{~d}$ and fixed by $4 \%$ PFA for $15 \mathrm{~min}$.

Real-time RT-PCR. RNA samples were extracted from netrin-1 wildtype and mutant spinal cords using RNeasy kit (Qiagen, Valencia, CA). RNA was pretreated with DNases to eliminate genomic DNA contaminations before making cDNAs by SuperScript first-strand cDNA synthesis kit (Invitrogen). Real-time PCR was performed on iCycler (Bio-Rad, Hercules, CA) using the relative expression method that same amount of cDNA was subjected to amplification of genes of interest and housekeeping gene glyceraldehyde-3-phosphate dehydrogenase (GAPDH). The difference of threshold cycles between the gene of interest and GAPDH from the same sample was defined as $\Delta C_{\mathrm{T}}$. Primer sequences used in this study can be distributed on request.

\section{Results \\ Lack of initial OPC dispersion in the netrin-1 mutant spinal cord}

Netrin-1 has been implicated in chick OPC dispersal in vitro (Tsai et al., 2003). To define the functions of netrin-1 in OPC migration in vivo, oligodendrocyte development was assayed in netrin-1 mutant mice. OPCs can be identified early in development through the expression of PDGF $\alpha$ R (Pringle and Richardson, 1993) and Olig2 transcription factor (Lu et al., 2000; Zhou et al., 2000). At E13.5, in both wild-type and netrin-1 mutant spinal cords, PDGF $\alpha$ R + cells were present at the ventricular zone; however, the dispersal and overall number of PDGF $\alpha \mathrm{R}+$ cells appeared to be substantially reduced in mutant animals (Fig. $1 A, B$ ). Olig2 + cells in E13.5 wild-type ventral spinal cord also showed extensive dispersal (Fig. 1C), whereas in the netrin-1 mutant spinal cord, Olig2 + cells were reduced in numbers and preferentially located at the ventricular zone (Fig. 1D). In all netrin-1 mutant spinal cords, evident perturbations of axonal projections, such as thinner ventral commissures, were seen, consistent with previous studies (Serafini et al., 1996).

To facilitate identification of spinal cord oligodendrocytes and their precursors, netrin-1 mutant mice were crossed into PLP-EGFP transgenic mice in which EGFP expression is mostly restricted to the oligodendrocyte cell lineage under the control of PLP promoter (Mallon et al., 2002). At E16, PLP-EGFP+ OPCs in the wild-type (Fig. $1 E$ ) and heterozygous (Fig. $1 F$ ) spinal cords were located at the ventral ventricular zone and dispersed into ventral white matter and occasionally into dorsal spinal cord. In 


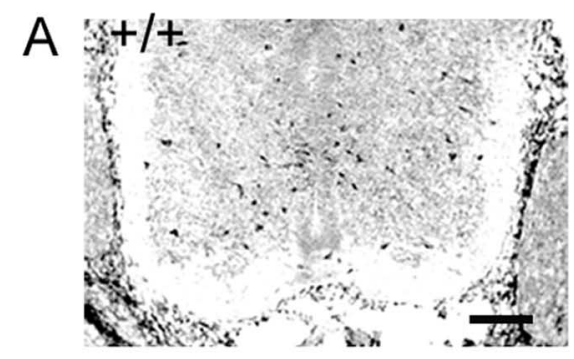

B
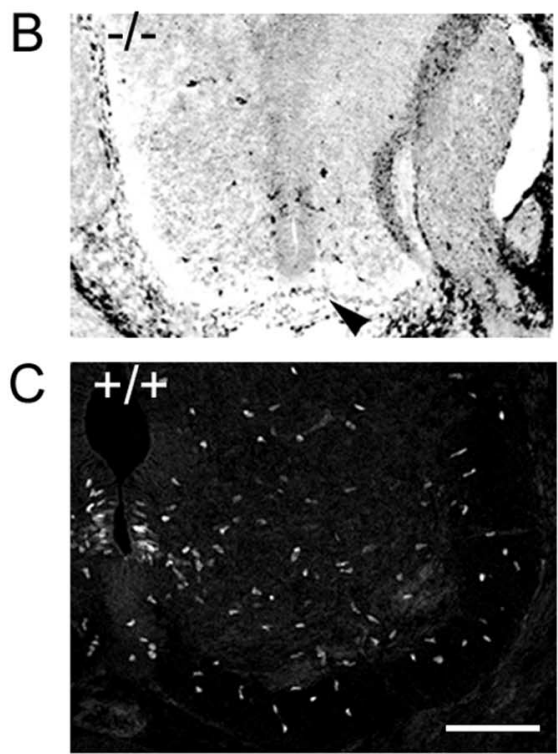

D

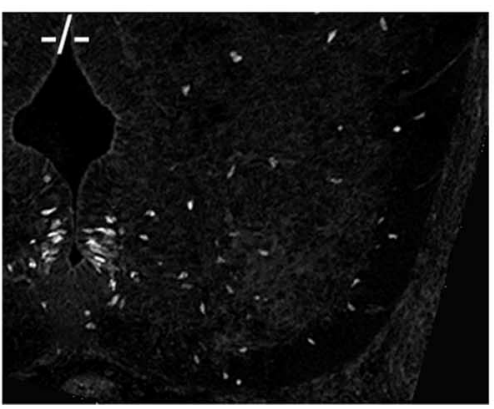

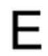
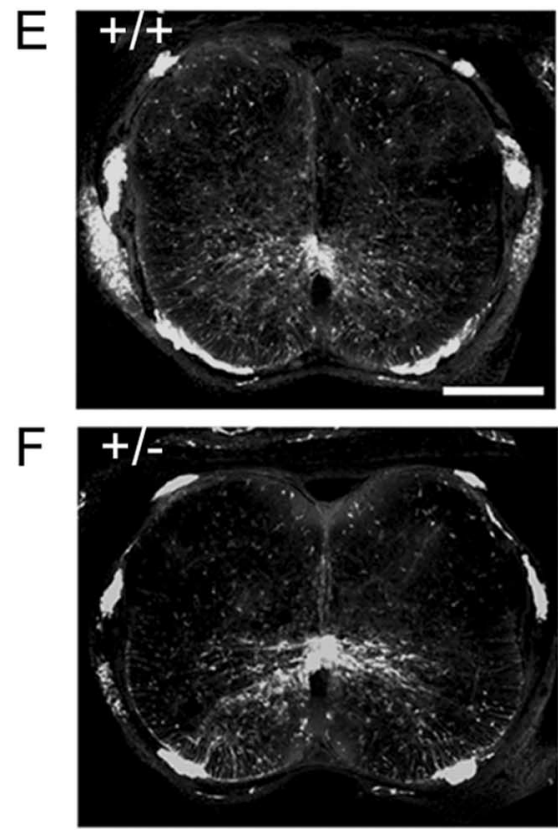

G

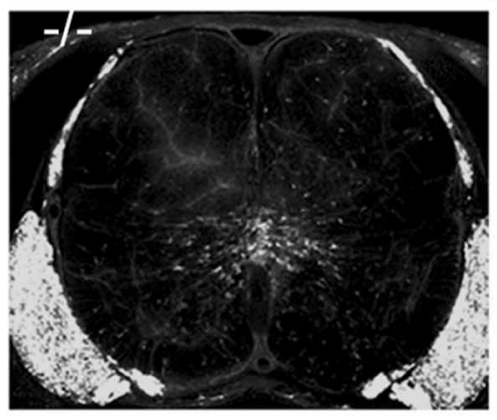

Figure 1. $\mathrm{OPC}$ dispersal is reduced in the netrin-1 mutant spinal cord. $\boldsymbol{A}, \boldsymbol{B}$, Expression of PDGF $\alpha$ R in the E13.5 spinal cord. $\operatorname{PDGF} \alpha \mathrm{R}+$ cells are widely dispersed in the ventral spinal cord $(\boldsymbol{A})$. In contrast, cells are less widely dispersed in the mutant spinal $\operatorname{cord}(\boldsymbol{B})$, and there is a reduction in cell number. Note that ventral commissures are thinner in mutant spinal cords because of the commissural axon guidance defect in netrin-1 mutants ( $\boldsymbol{B}$, arrowhead). $\boldsymbol{C}, \boldsymbol{D}$, Localization of 0lig2 + cells in the E13.5 spinal cord. 0lig2 + cells are widely dispersed in the wild-type spinal cord $(\boldsymbol{C})$ but more restricted to the ventral ventricular zone in mutant spinal cord (D). G-I, Distribution of PLP-EGFP + cells in the netrin-1 background. At E16, PLP-EGFP+ OPCs are dispersed throughout the ventral and dorsal lumbar spinal cords in wild-type $(\boldsymbol{E})$ and heterozygote $(\boldsymbol{F})$. In netrin-1 mutants, however, $0 P C S$ accumulate at the ventral ventricular zone $(\boldsymbol{G})$, and there is a reduction in cell number. Scale bars: $\boldsymbol{A}-\boldsymbol{D}, 100 \mu \mathrm{m} ; \boldsymbol{E}-\mathbf{G}, 250 \mu \mathrm{m}$.

contrast, in the netrin-1 mutant spinal cord, OPCs were clustered around the ventral ventricular zone with little dispersal to white matter (Fig. 1G). These data suggest that, in the mouse spinal cord, netrin-1 signaling is required for the dispersal of OPCs.

\section{Lack of directional migration of PLP-EGFP + OPCs in netrin-1 mutant}

Several different mechanisms may account for the limited dispersal of OPCs in netrin-1 mutant spinal cords, including a lack of migration, random migration, and differential survival. To directly assay the migrational behavior of OPCs in wild-type and mutant animals, time-lapse confocal imaging of living PLPEGFP+ cells was performed on E12.5 spinal cord slice cultures.
In wild-type slices, PLP-EGFP+ OPCs migrated radially from the ventricular zone toward the pial surface or the marginal zone (Fig. 2A) (video 1, available at www.jneurosci.org as supplemental material). Cells located close to the ventricular or intermediate zone established long processes toward the marginal zone, and the cell bodies moved rapidly along those processes. The majority of PLP-EGFP+ cells migrated directly away from the ventricular zone (tracings in Fig. 2 B) with some cells migrating as far as $100 \mu \mathrm{m}$ during a $3 \mathrm{~h}$ period. The rate of migration was not constant. Individual cells alternated periods of rapid migration with stationary intervals resulting in an overall saltatory migration much like that described previously for subventricular cells in the brain (Wichterle et al., 1997; Kakita and Goldman, 1999). In contrast, the migration of EGFP + cells in netrin-1 mutant spinal cords was extremely limited. Although the cells had a similar morphology with one long process extending toward presumptive white matter, the cell bodies of OPCs failed to undergo radial migration into the intermediate or marginal zones (Fig. 2C,D) (video 2, available at www.jneurosci.org as supplemental material). To establish the identity of PLP-EGFP+ cells as OPCs at E12.5, spinal cord sections from E12.5 PLPEGFP animals were labeled by Olig2 antibodies. A large proportion of PLP-EGFP+ cells in the ventral ventricular zone (Fig. $2 E$ ) and the intermediate zone (Fig. $2 F$ ) overlap with Olig2 + cells, consistent with the notion that the PLP-EGFP+ cells observed in living slices are OPCs.

OPC cell bodies in netrin-1 mutant spinal cords were not totally stationary; rather, they made small circular migratory paths in the ventricular zone, suggesting that the motile machinery was still intact in these cells. The lack of radial migration by OPCs in netrin-1 mutant animals may reflect either a lack of cell polarization or a reduction in the overall rate of nondirectional migration. To determine whether netrin- 1 affects the rate of migration, purified rat $\mathrm{A} 2 \mathrm{~B} 5+\mathrm{OPCs}$ were grown overnight in the presence or absence of netrin-1, and individual migration paths were recorded. In the absence of netrin-1, the basal migration rate was $0.48 \pm 0.03 \mu \mathrm{m} / \mathrm{min}(n=74)$, and this was not significantly altered by the addition of recombinant netrin-1 $(100 \mathrm{ng} / \mathrm{ml})(0.44 \pm 0.02 \mu \mathrm{m} / \mathrm{min} ; n=138 ; p=0.3)$. These data indicate that netrin-1 does not directly influence OPC chemokinesis in culture and imply that its primary role is to establish directional cues for migration.

\section{Reduction of OPC and differentiating oligodendrocyte cell numbers in netrin-1 mutants}

The number of oligodendrocytes and their precursors appeared to be substantially reduced in netrin-1 mutant spinal cords (Fig. 
1). If netrin-1 were a simple dispersal factor guiding OPC away from the ventricular surface, oligodendrocytes or their precursors might accumulate at their origin without a reduction in overall numbers. Comparison of numbers of precursors and oligodendrocytes in wild-type and netrin-1 mutant spinal cords revealed a widespread reduction at birth. The numbers of OPCs (NG2+ cells) (Fig. 3A,B), differentiating oligodendrocytes (O4+cells) (Fig. 3C,D), and mature oligodendrocytes (myelin basic protein mRNA+ cells) (Fig. $3 E, F)$ were all greatly reduced in mutant spinal cords. Furthermore, comparison of the number and distribution of mature oligodendrocytes in wild-type and netrin-1 mutant PLP-EGFP animals showed that there was a substantial reduction of peripherally located PLPEGFP + cells in the mutant ventral spinal cord (Fig. 3G,H). This apparent reduction in oligodendrocyte cell numbers was confirmed by the quantification of PLP-EGFP+ cells (Fig. 3I). The number of EGFP + cells in wild-type ventral spinal cord per longitudinal section was $64 \pm 12.14$, approximately twice the number of EGFP + cells in the mutant ventral spinal cord $(31.71 \pm 5.5$ cells/ section; $p=0.03$ ). In the dorsal spinal cord, whereas wild-type sections contained $13.57 \pm 5.96$ cells/section, netrin- 1 mutant animals had only $0.11 \pm 0.07$ cells/section $(p=0.02)$. No significant statistical difference between wild-type and heterozygous animals were found. Together, these studies suggest that OPCs differentiate on a normal schedule in the netrin-1 mutant spinal cord, but their numbers are reduced throughout development.

The reduction in PLP-EGFP cell number was widespread in the CNS of netrin-1 mutant animals. Early differentiating OPCs in the hindbrain arise in a narrow region along the midline of the pons and medulla (Hardy and Friedrich, 1996). In sagittal sections of wild-type brain (Fig. 3L), one group of PLP-EGFP + cells was located caudally at the level of the cerebellum and medulla, whereas another was located more rostrally in the diencephalon. In the absence of netrin-1, the number of PLP-EGFP+ cells was substantially reduced in both groups (Fig. $3 M$ ).

Not all neural lineages were affected by the lack of netrin-1. Neurofilament staining on mutant spinal cord sections showed aberrant axonal patterns consistent with previous reports (Serafini et al., 1996), and the number of oligodendrocyte lineage cells was reduced. In contrast, the density and distribution of GFAP+ astrocytes did not appear to be substantially different between wildtype and mutant animals (Fig. $3 \mathrm{~J}, \mathrm{~K}$ ). In both cases, spinal cord white matter astrocytes were primarily radially oriented. These data suggest that the absence of netrin-1 primarily affects neuronal and oligodendrocyte populations in the developing CNS.

\section{Netrin-1 is not essential for oligodendrocyte development in vitro}

In other systems, it has been suggested that the netrin receptors DCC and UNC5 are dependence receptors and, when unoccu- pied by ligands, may induce cell death by apoptosis (Mehlen et al., 1998). Oligodendrocyte lineage cells are known to be susceptible to apoptotic cell death (Calver et al., 1998), and such a mechanism may account for their reduced numbers in netrin-1 mutants. To determine whether OPC survival is regulated by netrin-1, A2B5+ and O4+ OPCs purified by immunopanning were cultured for $3 \mathrm{~d}$ in the presence of recombinant netrin- 1 or anti-DCC antibodies, and survival was measured by MTT cell viability assays. Cells grown in DMEM alone served as negative controls, whereas cells grown in complete medium served as positive controls and were normalized to $100 \%$. Neither the addition of recombinant mouse netrin-1 or the presence of anti-DCC antibodies altered A2B5+ or O4+ cell survival significantly (Fig. $4 A$ ), suggesting that in the context of oligodendrocytes, netrin-1 signaling is not required for survival. To more clearly define the role of netrin- 1 in spinal cord oligodendrogenesis, the capacity of normal and mutant ventral spinal cord explants to generate oligodendrocytes was compared. In E13-derived explants, a slight reduction in the number of $\mathrm{O} 4+$ cells that developed in mutant explants (287.2 \pm 37.97 cells/explant) was seen after $3 \mathrm{~d}$ in vitro compared with wild-type explants ( $429.25 \pm 68.3$ cells/explant) (Fig. 4B). Parallel explants derived from E16 embryos demon- 


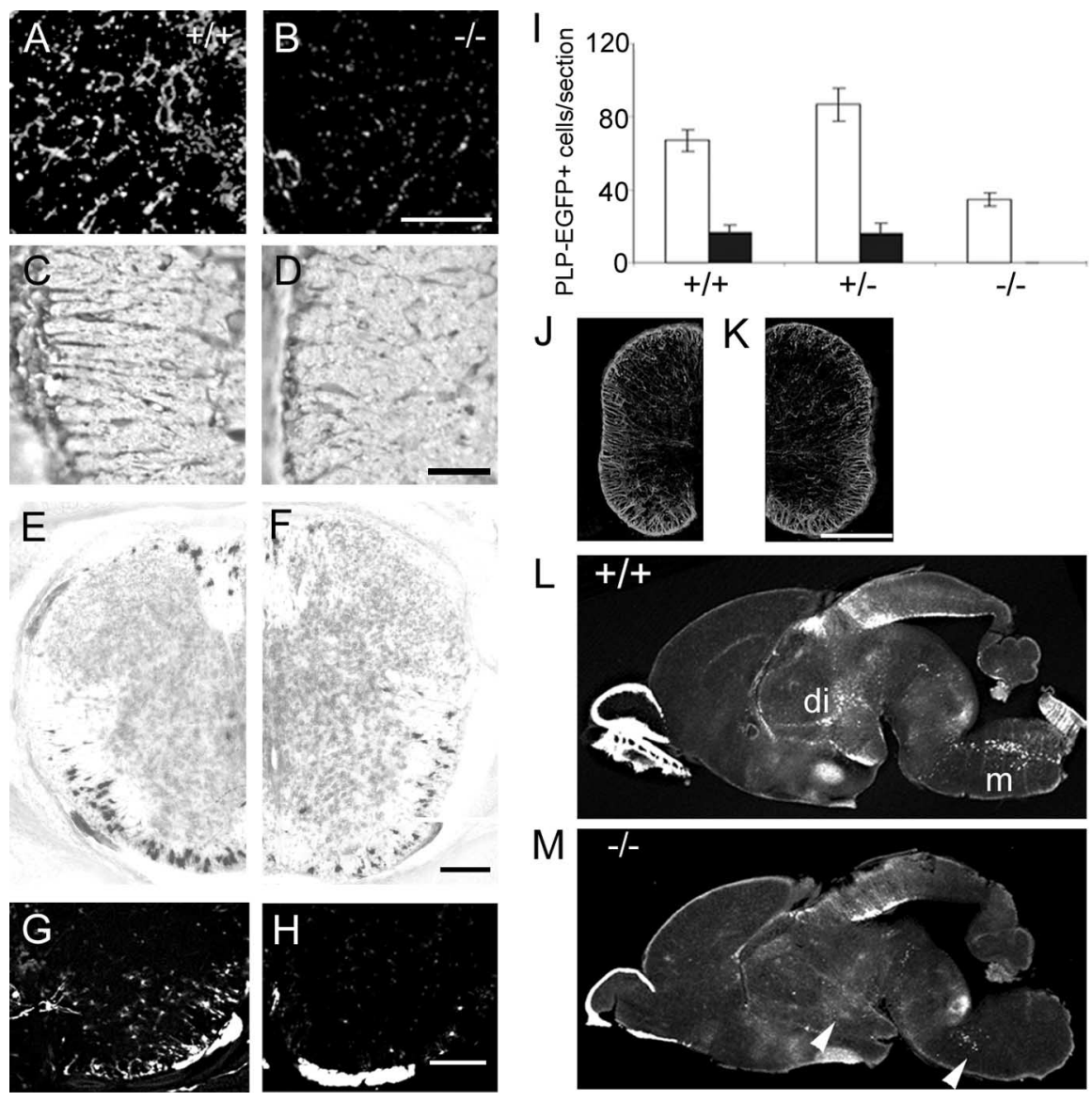

Figure 3. Analysis of oligodendrocyte development at P0. Oligodendrocyte markers were used to examine wild-type and netrin-1 mutant spinal cord sections. $\boldsymbol{A}, \boldsymbol{B}, \mathrm{NG} 2$ expression is lower in the lateral funiculus of the mutant $(\boldsymbol{B})$ than in wild-type spinal cords $(\boldsymbol{A}) . \boldsymbol{C}, \boldsymbol{D}$, Fewer $04+$ cells are present in the lateral white matter of the mutant $(\boldsymbol{D})$ than in wild-type spinal cords (C). $\boldsymbol{E}, \boldsymbol{F}$, In situ hybridization for MBP shows that oligodendrocytes are present in the ventral and dorsal white matter in the wild-type $(\boldsymbol{E})$ spinal cord at P0.F, In the mutant spinal cord, fewer MBP + cells are present in the ventral white matter, and only rare cells are shown in the lateral and dorsal white matter. G-J,PLP-EGFP + cells in lumbar spinal cord sections. Consistent with MBP labeling, bright PLP-EGFP + cells are present in the ventral funiculus in the wild-type $(\boldsymbol{G})$ but greatly reduced in the mutant spinal cord $(\boldsymbol{H})$. $I$, Quantification of PLP-EGFP + cells in longitudinal spinal cord sections confirms the reduction of cell numbers in mutant spinal cords. Open bars indicate ventral spinal cords, and solid bars indicate dorsal spinal cords. $\boldsymbol{J}, \boldsymbol{K}$, The distribution of astrocytes is not obviously different between wild type and the mutant shown by GFAP staining. $L, M$, Oligodendrocyte developmental defect is also present in the netrin-1 mutant brain. $L$, Sagittal section of wild-type brain shows that there are two prominent groups of PLP-EGFP + cells present in the diencephalon (di) and medulla $(\mathrm{m}) . M$, PLP-EGFP + cells are nearly absent in those two regions in the mutant brain shown by arrowheads. Scale bars: $\boldsymbol{A}-\boldsymbol{D}, 50 \mu \mathrm{m} ; \boldsymbol{E}-\boldsymbol{H}, 100 \mu \mathrm{m} ; \boldsymbol{J}, \boldsymbol{K}, 250 \mu \mathrm{m}$. Error bars represent SEM.

The local environment of netrin-1 mutant spinal cords does not promote normal oligodendrocyte development One explanation for the failure of oligodendrocytes to thrive in netrin-1 mutant spinal cords is that dispersal into the spinal cord parenchyma is required to receive appropriate cues. For example, germ cells in the mouse arise from extraembryonic origins and migrate long distances to the somatic gonadal cells to form the embryonic gonad. Successful proliferation of germ cells is dependent on this migration, because germ cell mitogens are expressed along the specific migratory path (StarzGaiano and Lehmann, 2001). To test this hypothesis, ventral spinal cord explants from netrin-1 mutant animals were labeled and transplanted to more lateral regions in wild-type spinal cord slices. Labeled cells migrated out of mutant explants toward the pial surface in wildtype slices (data not shown). In contrast, little or no cell migration was seen when wild-type or mutant explants were transplanted into mutant slices. These observations suggest that both mutant and wildtype neural cells can respond to netrin cues at a distance from the midline and that wild-type but not netrin-1 mutant slices support neural cells and their dispersal. Transplanted ventral explants contained multiple cell types, and to selectively assay the behavior of OPCs, PLP-EGFP+ cells were transplanted into wild-type or mutant spinal cord slices. When injected close to the ventral or dorsal midlines, PLPEGFP + cells showed extensive radial migration in wild-type slices (Fig. $5 A, C, E$ ). This was particularly evident at the ventral midline, where cells migrated selectively toward the peripheral white matter and rarely crossed the midline (Fig. $5 C$ ). Injected cells remained process-bearing during the culture period (Fig. $5 F$ ), indicating that their viability was supported by the

strated a more pronounced reduction of $\mathrm{O} 4+$ cells in netrin-1 mutant $(41.67 \pm 14.99$ cells/explant $)$ than wild-type explants (169.08 \pm 13.56 cells/explant). The relative reduction of OPC numbers was progressive through development, and by $\mathrm{P} 0$, there were $122 \pm 20.58$ cells/explant from wild-type spinal cords but only $9.75 \pm 2.11$ cells/explant from the mutant spinal cord. The developmentally associated loss of oligodendrocyte lineage cells in the netrin-1 mutant spinal cord was dependent on the environment. E16 mutant explants maintained for $5 \mathrm{~d}$ in culture, $\mathrm{O} 4+$ cell numbers $(258.66 \pm 30.14$ cells/explant) began to normalize to those in wild-type explants $(344.38 \pm 37.72$ cells/explant). Together, these data indicate that netrin- 1 signaling is not essential for the survival or development of isolated OPCs, and that the progressive loss of oligodendrocytes in the mutant spinal cord reflects the inability of cells to mature through critical stages because of inappropriate localization or misregulated environmental cues in vivo. wild-type environment. In contrast, in netrin-1 mutant spinal cords, injected cells failed to disperse from the injection site (Fig. $5 B, D)$, and the number of labeled cells $(6.19 \pm 3.85$ cells/slice; $n=22$ ) did not increase noticeably as in the wild types (64.56 \pm 14.78 cells/slice; $n=16 ; p=0.001$ ). To unambiguously follow the behavior of OPCs and to rule out the possibility that the PLP promoter was differentially regulated in the absence of netrin-1, the injection paradigm was repeated with immature highly migratory pan-purified rat A2B5+ cells. A similar result was observed with less dispersal and fewer cells $(6.83 \pm 2.52$ cells/mutant slice; $n=23$ ) present in the mutant slices compared with wild-type and heterozygous slices $(80.18 \pm 17.98$ cells/slice; $n=$ $11 ; p<0.001$ ) (Fig. $5 G-J$ ), although slightly more dispersal was seen with purified cells than with PLP-EGFP+ cells (compare Fig. 5, $A$ and $G$ ). This likely reflects the fact that PLP-EGFP+ cells represent multiple stages of oligodendrocyte development and not exclusively early migratory cells. Consistent with this hypoth- 


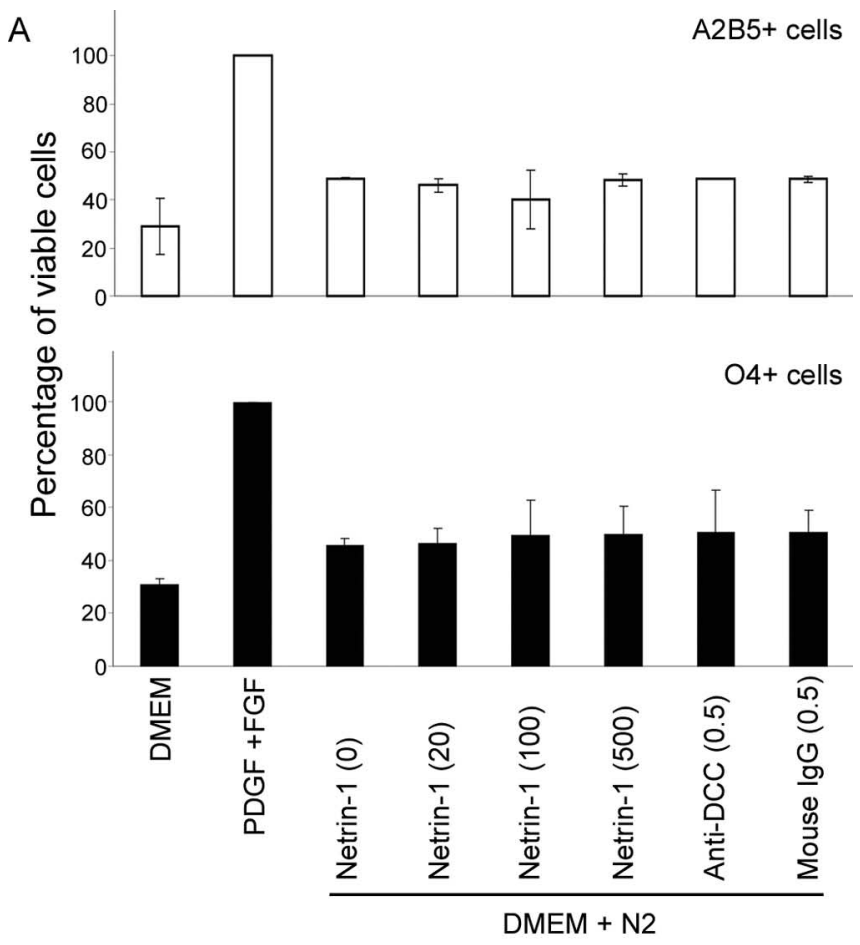

B

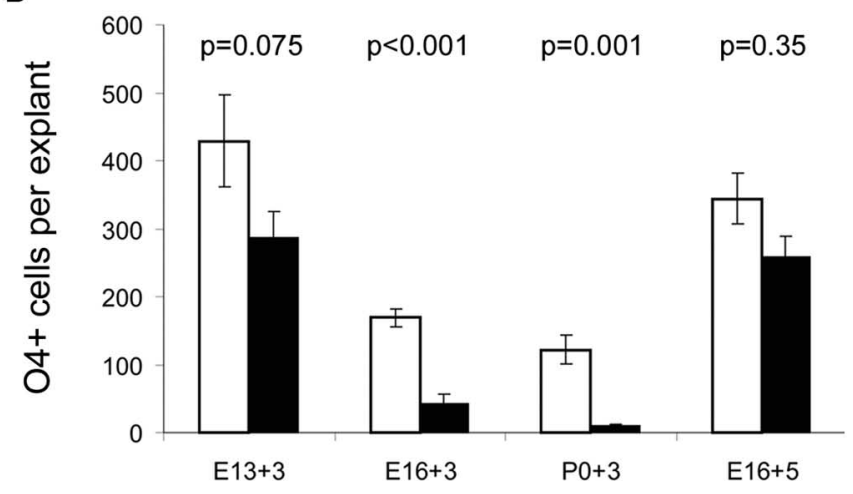

Figure 4. $\quad O P C$ survival in vitro is not dependent on netrin-1. A, Purified $\mathrm{A} 2 \mathrm{~B} 5+$ and $04+$ OPCs were grown under various conditions, and cell survival was measured by MTT assays. The cell survival of OPCs in the complete medium with PDGF and basic FGF is normalized at $100 \%$, whereas the cell survival in DMEM-only medium is considered the negative control. The presence of netrin-1 (at $\mathrm{ng} / \mathrm{ml}$ indicated in parentheses) in the minimal medium (DMEM with N2 supplement) does not significantly promote cell survival, regardless of the concentration. In addition, anti-DCC antibodies for blocking netrin-1 signaling as well as control mouse lgG antibodies used at $0.5 \mu \mathrm{g} / \mathrm{ml}$ do not significantly increase cell death. $\boldsymbol{B}$, Ventral spinal cord explants were dissected and cultured from litters of different ages. The relative number of $04+$ cells present in the mutant explants (solid bars) is progressively reduced during embryonic development compared with wild-type explants (open bars). Prolonged cultures in permissive environments allow mutant explants to mostly recover (see E16 +5 d). Error bars represent SEM.

esis, PLP-EGFP + cells had both bipolar and myelinating morphologies (Fig. 5F). Together, these studies suggest that the environment of the netrin-1 mutant spinal cord is not conducive to either oligodendrocyte dispersal or expansion.

\section{Proliferation, but not cell death, is altered in the netrin-1 mutant spinal cord}

The failure of expansion of endogenous or transplanted OPCs in the netrin-1 mutant spinal cord may reflect reduced cell proliferation or increased cell death. There was a consistent reduction of BrdU + cells in the netrin- 1 mutant animals after a $16 \mathrm{~h}$ pulse at
E16, whereas many proliferating cells were present in wild-type spinal cord white matter (Fig. $6 A, B$ ). To extend these studies and confirm the cell identity, spinal cord cell cultures were established from wild-type and mutant animals labeled by BrdU for $2 \mathrm{~h}$ in vivo before harvesting. After $15 \mathrm{~h}$ in culture, cells were labeled with anti-NG2 and anti-BrdU antibodies. A significant reduction $(p=0.003)$ in the proportion of BrdU+ cells within the total $\mathrm{NG} 2+$ cell population was seen in mutant $(18 \pm 3 \%)$ compared with wild-type $(32 \pm 3 \%)$ spinal cord cultures, suggesting that the proliferation of OPCs was significantly compromised in netrin mutant spinal cords. In contrast, cell death assayed by the comparison of the numbers of activated caspase-3+ or terminal deoxynucleotidyl transferase-mediated biotinylated UTP nick end labeling (TUNEL) ${ }^{+}$cells in wild-type and mutant spinal cords revealed no significant differences (Fig. 6C). Because oligodendrocyte development is dependent on environment-derived factors such as PDGF-AA, we analyzed cytokine expression by real-time PCR to determine whether lower levels of oligodendrocyte proliferation reflected lower levels of cytokine expression. No global changes in the expression of PDGF-AA or the neuregulin gene family (Meyer et al., 1997; Vartanian et al., 1999) were found (Table 1). A marginal reduction of EphA2 receptor expression was present in netrin-1 mutant animals that might reflect the pathfinding defect of commissural neurons (Brittis et al., 2002). These data imply that reduced proliferation in OPCs is responsible for the reduction of cell numbers in netrin-1 mutants and that the reduction in OPC proliferation is a consequence of changes in the local cytological environment of the white matter rather than global changes in growth factor expression.

\section{Discussion}

Netrin-1 has been implicated in guiding cell migration and axonal pathfinding in a variety of systems. Here, we show that, in the murine spinal cord, it is a potent dispersal factor for OPCs. Time-lapse analyses of EGFP-labeled cells in wild-type and netrin-1 mutant spinal cords demonstrated that in the absence of netrin-1, OPCs were unable to migrate radially away from the ventricular zone at the onset of dispersal and subsequently were less widely distributed in the mutant spinal cord. Concomitant with the reduction in OPC dispersal was a developmentally progressive loss of oligodendrocytes in the netrin-1 mutants. Although netrin-1 clearly mediates OPC dispersal, the reduction in oligodendrocyte lineage cell number is not a consequence of netrin-1-mediated survival or proliferation. In vitro, addition of netrin-1 did not promote purified OPC survival or proliferation. Likewise, exposure of cells to function-blocking anti-DCC had no detectable effect. Overcoming the initial dispersal defect by transplanting cells to presumptive white matter did not reverse OPC loss. OPCs injected into wild-type hosts dispersed and survived effectively, whereas the same cells injected into netrin-1 mutant hosts failed to thrive. We propose that netrin- 1 is essential for both OPC dispersal from the ventricular zone and establishing a white matter environment that supports the subsequent elaboration of a normal number of oligodendrocytes.

\section{Netrin-1 mediates cell dispersal by promoting cell polarity}

Netrin-1 is an effective chemorepellent for OPCs in the intact embryonic spinal cord. Previous in vitro data suggested that netrin-1-mediated chemorepulsion in OPCs and the present studies provide strong support for this hypothesis in vivo. The requirement of netrin-1 in OPC directional migration is evident from the fact that OPCs fail to migrate in netrin-1 mutant slices, although netrin-1 does not affect general OPC chemokinesis. In 


\section{PLP-EGFP+ cells}
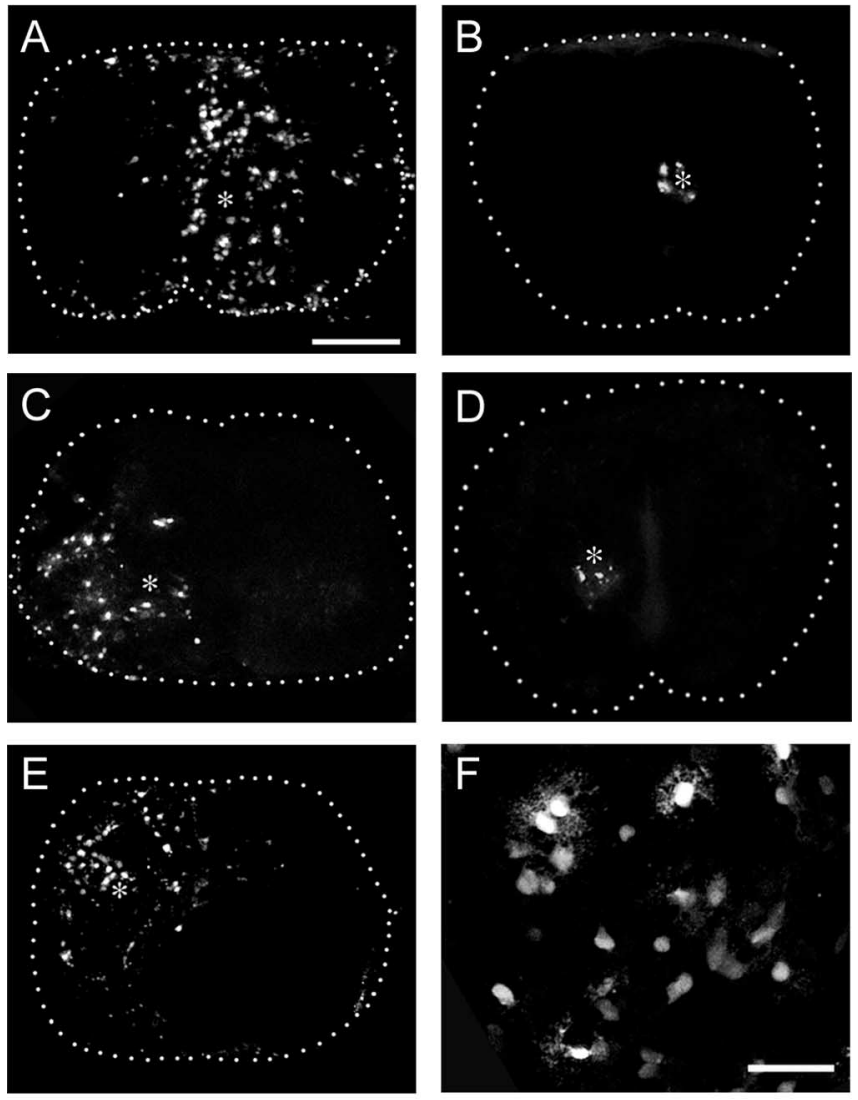

A2B5+ cells
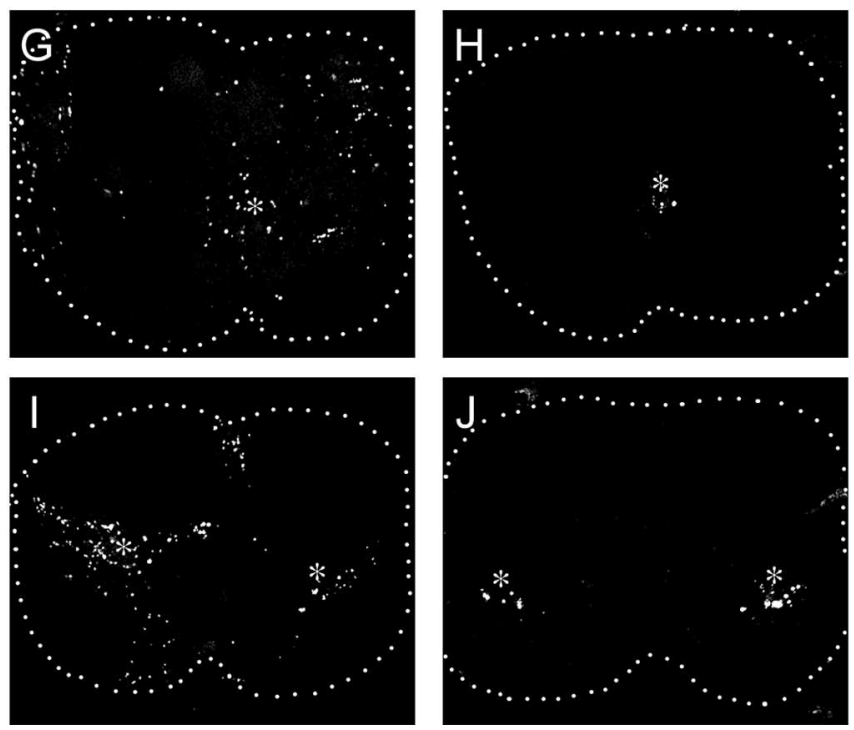

Figure 5. Netrin-1 is a strong dispersal factor for $\mathrm{OPCS} A, C, E, F$, E18 wild-type spinal cord slices. $\boldsymbol{B}, \boldsymbol{D}$, E18 netrin-1 mutant spinal cord slices. $\boldsymbol{A}, \boldsymbol{B}$, Injection of PLP-EGFP+ cells into the central canal region. In wild-type slices $(\boldsymbol{A})$, PLP-EGFP+ cells disperse radially from the injection site. The cells are healthy and retain their morphology within the slice $(\boldsymbol{F})$. In netrin-1 mutant slices, PLP-EGFP + cells remain close to the injection sites $(\boldsymbol{B})$. Note that there are fewer cells in $\boldsymbol{B}$ than in $\boldsymbol{A}$. $\boldsymbol{C}, \boldsymbol{D}$, Injection of PLP-EGFP + cells into ventral spinal cords. PLP-EGFP + cells injected into wild-type ventral spinal cord disperse as a wedge from the injection site with the majority of cells migrating toward the white matter. The cells do not cross the midline (C).D, In contrast, no dispersal is seen after injection into mutant ventral spinal cords. $\boldsymbol{E}$, Injection of PLP-EGFP + cells into dorsal spinal cord results in a wedge shape migration to the white matter mirroring the ventral spinal cord. Asterisks indicate injection sites. Scale bars: $\boldsymbol{A}-\boldsymbol{E}, 250 \mu \mathrm{m} ; \boldsymbol{F}$, $50 \mu \mathrm{m}$. To rule out the possibility that the reduction of PLP-EGFP + cells in netrin-1 mutant
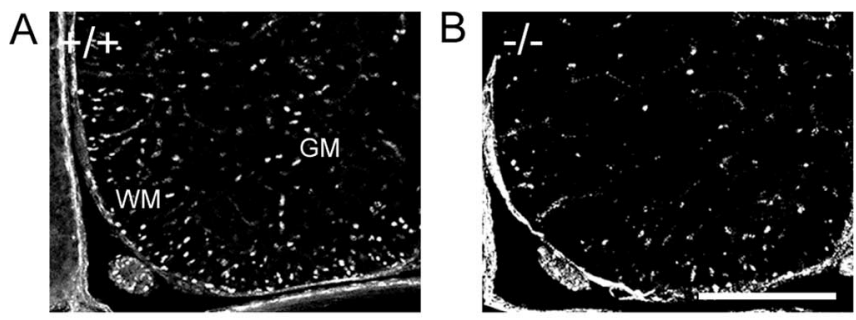

C

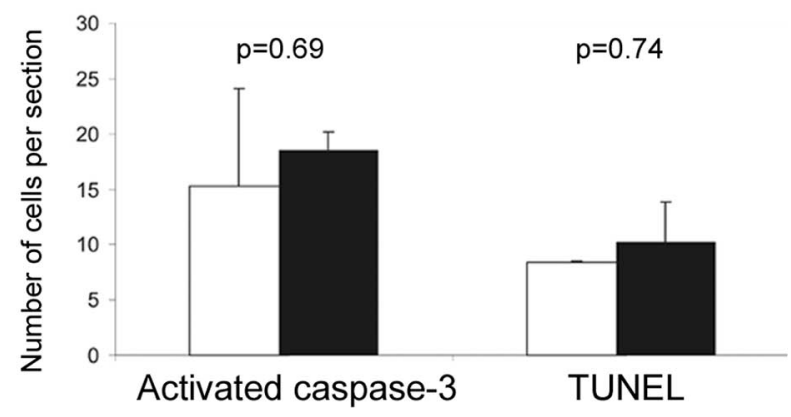

Figure 6. Netrin-1 mutant spinal cords show a reduction in cell proliferation but not cell death. $\boldsymbol{A}$, Ventral spinal cord sections from wild-type animals show proliferating cells labeled by anti-BrdU antibodies in both gray matter (GM) and white matter (WM). $\boldsymbol{B}$, In netrin-1 mutant spinal cord, the number of proliferating cells is dramatically reduced in both gray matter and white matter. C, Cell death detected by antibodies to activated caspase-3 or TUNEL method while slightly increased, it is not significantly altered in the E17 mutant spinal cord (solid bars) compared with wild type (open bars). Scale bar: $A, B, 250 \mu \mathrm{m}$. Error bars represent SEM.

Table 1. Expression of selected growth factor mRNAs in netrin-1 wild-type and mutant animals detected by real-time RT-PCR

\begin{tabular}{lllll}
\hline & \multicolumn{4}{c}{$\Delta C_{\mathrm{T}_{\text {gene efinterst-GAPH }}{ }^{a}}$} \\
\cline { 2 - 5 } & PDGF-AA & Neuregulin-1 & Neuregulin-3 & EphA2 \\
\hline$+/+(n=5)$ & $2.72 \pm 0.2$ & $4.87 \pm 0.19$ & $5.49 \pm 0.34$ & $11.05 \pm 0.18$ \\
$-/-(n=7)$ & $2.73 \pm 0.39$ & $4.99 \pm 0.45$ & $5.5 \pm 0.37$ & $10.63 \pm 0.5$ \\
vvalue $^{b}$ & 0.97 & 0.6 & 0.94 & 0.1
\end{tabular}

${ }^{a} \Delta C_{T}$ is defined as the threshold cycle difference between the gene of interest and the housekeeping gene GAPDH. The data are expressed as mean \pm SD.

${ }^{b}$ Three litters of netrin- 1 animals were analyzed for the expression of genes listed above using a $t$ test. In no cases were significant differences in the expression of the selected growth factor identified.

wild-type hosts, transplanted cells rarely migrated toward or crossed the midline where the expression of netrin-1 is highest (Kennedy et al., 1994). Rather, cells preferentially migrated toward the pial surface even if transplanted in relatively lateral locations. This suggests that netrin-1 is capable of exerting positional information extending across the entire tissue. How far netrin-1 can freely diffuse within tissues is still unclear (Manitt and Kennedy, 2002). In Drosophila, netrin mRNA and protein distributions in the nerve cord are not colocalized, and it has been proposed that netrin proteins are captured and immobilized by axons expressing netrin receptors distal from netrin-secreting cells as a presentation for other netrin-guided axons (Hiramoto et al., 2000). In this model, OPCs transplanted at relatively lateral locations in the wild-type spinal cord can still be guided by

$\longleftarrow$

spinal cords was attributable to the differential regulation of the PLP promoter, the injection experiment was repeated using labeled rat $A 2 B 5+$ cells. $\boldsymbol{G}, \boldsymbol{H}$, Injection of labeled $A 2 B 5+$ cells into the central canal region. $\mathbf{G}, A 2 B 5+$ cells in the wild-type slice migrate radially from the injection site, whereas they remain localized around the injection site in netrin-1 mutant slices $(\boldsymbol{H})$. When cells were injected into the ventral spinal cord, they migrate toward the white matter in the wild-type slice $(\boldsymbol{I})$ but fail to do so in the mutant slice $(\boldsymbol{J})$. Asterisks indicate injection sites. Scale bar: $\mathbf{G}-\mathbf{J}, 250 \mu \mathrm{m}$. 
netrin-1. Such a model is not restricted to the vertebrate neural tube. In grasshopper limbs, a continuous tissue gradient that can be interpreted by developing pioneering axons has been proposed (Lefcort and Bentley, 1987).

In both wild-type and mutant spinal cords, the earliest oligodendrocyte lineage cells extend processes toward the pial surface while retaining the cell body at the ventricular surface. OPC cell bodies in wild-type environments migrated primarily unidirectionally away from the ventricular zone but in netrin-1 mutant environments oscillated back and forth within the ventricular zone. These observations suggest that directed cell soma movements rather than overall morphological cell polarity is dependent on netrin-1 signaling. A lack of directed cell soma movement would contribute to the lack of overall motility seen after transplantation to lateral spinal cord regions. How netrinmediated cues are integrated into directional movements is unknown, although several relevant proteins have been shown to tightly associate with netrin-1 signaling in growth cones. For example, small GTPases important in establishing cell polarity are known to be activated by netrin-1 signal (Shekarabi and Kennedy, 2002) as well as focal adhesion kinase and src family kinases (Li et al., 2004; Ren et al., 2004), and similar mechanisms are likely to mediate directed migration of OPCs.

In addition to netrin-1, several other molecular regulators of OPC migration have been proposed, including semaphorins (Spassky et al., 2002; Cohen et al., 2003) as well as Eph-ephrin interactions that might regulate optic nerve OPC migration (Prestoz et al., 2004). Currently, nothing is known about the expression of these other guidance cues in the absence of netrin, and they might be misexpressed or provide conflicting guidance information. It is not clear whether all oligodendrocytes respond similarly to netrin cues. Recent studies suggest that oligodendrocytes are derived from multiple origins (Cai et al., 2005; Vallstedt et al., 2005), and distinct pools of cells may have distinct responses, which would account for their different migratory behaviors. Likewise, even within a single pool of cells, responses to netrin may change depending on the stage of OPC development (Spassky et al., 2002). Such flexibility may allow for more precise positioning of cells as development proceeds.

\section{Generation of correct oligodendrocyte numbers}

During normal development, oligodendrocyte numbers are closely matched with the number of axons to be myelinated (Barres et al., 1992), and this appears to be disrupted in the absence of netrin-1. Several mechanisms regulate the generation of appropriate numbers of oligodendrocytes. In general, excess OPCs are produced, and the final number is established later by apoptosis (Barres et al., 1992; Calver et al., 1998). In the absence of netrin-1, the current data suggest that proliferation is impaired, resulting in insufficient OPCs at the early stage of oligodendrocyte development, and there is no evidence of an elevation in cell death. One of the major mitogens for OPCs is PDGF, and overexpression of PDGF results in excess OPCs, whereas reductions in PDGF result in reduced numbers of OPCs (Calver et al., 1998; Fruttiger et al., 1999). Furthermore, detailed analyses of OPC proliferation in vivo under different levels of mitogen stimulation demonstrate that the final cell numbers are a reflection of mitogen supply and demand (van Heyningen et al., 2001). Although OPC proliferation was reduced in netrin-1 mutants, no obvious reduction in PDGF was detected by real-time PCR, suggesting that the regulation of OPC proliferation is not simply controlled by global levels of PDGF. Consistent with the supply and demand model, however, the failure of OPCs to migrate and disperse in the absence of netrin-1 would result in a local high-cell density and elevated growth factor demand with a consequent suppression of proliferation through competition for local growth factors. Such a model may also underlie a densitydependent feedback mechanism (Zhang and Miller, 1996) that contributes to the reduction in proliferation of OPCs in netrin-1 mutants. Indeed, in other systems, a functional link between decreased cell motility and decreased cell proliferation has been proposed (Gilmore and Romer, 1996).

Not only soluble growth factors but also the intact cytoarchitecture of the spinal cord is critical for oligodendrocyte development. For example, OPCs are transiently severely reduced in Mash1 and Math3 double mutants with altered cytoarchitecture in the midline because of aberrant neural progenitor phenotypes in the hindbrain (Ohsawa et al., 2005). Multiple axonal tracts are affected in netrin-1 mutants, including inhibitory GABAergic commissural and engrailed-1+ (En-1) spinal interneurons with pathfinding errors and short axonal outgrowth (Serafini et al., 1996; Saueressig et al., 1999). Oligodendrocyte development can be regulated by a variety of neurotransmitters (Gallo et al., 1996), and direct synaptic signaling between GABAergic interneurons and OPCs in the hippocampus regulates oligodendrocyte development (Lin and Bergles, 2004). Therefore, a subpopulation of interneurons with perturbed trajectories in netrin-1 mutants may secondarily affect oligodendrocyte development. Finally, multiple molecular signals such as neurotrophins and chemokines influence OPC proliferation and survival, and the expression of these cues is likely to be dependent on correct white matter cytoarchitecture.

Interestingly, the lack of increased OPC cell death in netrin-1 mutant contrasts with recent findings, suggesting that netrin-1 signaling mediates a preapoptotic pathway. The netrin receptors DCC and Unc5 have been proposed as dependence receptors, such that the absence of ligand engagement with the cell surface receptor induces apoptosis (Mehlen et al., 1998; Llambi et al., 2001). For example, DCC or Unc5-expressing precerebellar neurons undergo massive cell death in netrin-1 mutants (Llambi et al., 2001), whereas overexpression of netrin-1 in the mouse gastrointestinal tract inhibited intestinal cell apoptosis and promoted hyperplasia and adenoma in the intestine (Mazelin et al., 2004). The latter has been implicated in colorectal tumorigenesis. The lack of effect of netrin-1 on OPC survival suggests that netrin and netrin receptors mediate different intracellular signaling pathways in different cell types. For example, in mammary gland morphogenesis, netrin-1 acts as a local adhesive signal to organize the multipotent progenitor cell layer instead of being a guidance molecule (Srinivasan et al., 2003).

The current findings suggest proper oligodendrocyte development is dependent on multiple signals and an intact environment. The dependence of OPC expansion on netrin-regulated motility suggest that successful repair of demyelinated lesions in the adult CNS will not simply be accomplished by elevation of growth or survival factor concentrations but will be critically dependent on the dispersal of newly generated proliferating progenitors. Identification of the repertoire of required signals will likely yield novel therapeutic approaches.

\section{References}

Barres BA, Raff MC (1999) Axonal control of oligodendrocyte development. J Cell Biol 147:1123-1128.

Barres BA, Hart IK, Coles HS, Burne JF, Voyvodic JT, Richardson WD, Raff MC (1992) Cell death and control of cell survival in the oligodendrocyte lineage. Cell 70:31-46. 
Bhat MA (2003) Molecular organization of axo-glial junctions. Curr Opin Neurobiol 13:552-559.

Brittis PA, Lu Q, Flanagan JG (2002) Axonal protein synthesis provides a mechanism for localized regulation at an intermediate target. Cell 110:223-235.

Cai J, Qi Y, Hu X, Tan M, Liu Z, Zhang J, Li Q, Sander M, Qiu M (2005) Generation of oligodendrocyte precursor cells from mouse dorsal spinal cord independent of Nkx6 regulation and Shh signaling. Neuron 45:41-53.

Calver AR, Hall AC, Yu WP, Walsh FS, Heath JK, Betsholtz C, Richardson WD (1998) Oligodendrocyte population dynamics and the role of PDGF in vivo. Neuron 20:869-882.

Cohen RI, Rottkamp DM, Maric D, Barker JL, Hudson LD (2003) A role for semaphorins and neuropilins in oligodendrocyte guidance. J Neurochem 85:1262-1278.

Freeman MR, Delrow J, Kim J, Johnson E, Doe CQ (2003) Unwrapping glial biology: $\mathrm{Gcm}$ target genes regulating glial development, diversification, and function. Neuron 38:567-580.

Fruttiger M, Karlsson L, Hall AC, Abramsson A, Calver AR, Bostrom H, Willetts K, Bertold CH, Heath JK, Betsholtz C, Richardson WD (1999) Defective oligodendrocyte development and severe hypomyelination in PDGF-A knockout mice. Development 126:457-467.

Gallo V, Zhou JM, McBain CJ, Wright P, Knutson PL, Armstrong RC (1996) Oligodendrocyte progenitor cell proliferation and lineage progression are regulated by glutamate receptor-mediated $\mathrm{K}^{+}$channel block. J Neurosci $16: 2659-2670$.

Gilmore AP, Romer LH (1996) Inhibition of focal adhesion kinase (FAK) signaling in focal adhesions decreases cell motility and proliferation. Mol Biol Cell 7:1209-1224.

Guan KL, Rao Y (2003) Signalling mechanisms mediating neuronal responses to guidance cues. Nat Rev Neurosci 4:941-956.

Hardy RJ, Friedrich VL Jr (1996) Oligodendrocyte progenitors are generated throughout the embryonic mouse brain, but differentiate in restricted foci. Development 122:2059-2069.

Hatten ME (2002) New directions in neuronal migration. Science 297:1660-1663

Hiramoto M, Hiromi Y, Giniger E, Hotta Y (2000) The Drosophila Netrin receptor Frazzled guides axons by controlling Netrin distribution. Nature 406:886-889.

Hong K, Hinck L, Nishiyama M, Poo MM, Tessier-Lavigne M, Stein E (1999) A ligand-gated association between cytoplasmic domains of UNC5 and DCC family receptors converts netrin-induced growth cone attraction to repulsion. Cell 97:927-941.

Jarjour AA, Kennedy TE (2004) Oligodendrocyte precursors on the move: mechanisms directing migration. Neuroscientist 10:99-105.

Jarjour AA, Manitt C, Moore SW, Thompson KM, Yuh SJ, Kennedy TE (2003) Netrin-1 is a chemorepellent for oligodendrocyte precursor cells in the embryonic spinal cord. J Neurosci 23:3735-3744.

Kakita A, Goldman JE (1999) Patterns and dynamics of SVZ cell migration in the postnatal forebrain: monitoring living progenitors in slice preparations. Neuron 23:461-472.

Keino-Masu K, Masu M, Hinck L, Leonardo ED, Chan SS, Culotti JG, TessierLavigne M (1996) Deleted in colorectal cancer (DCC) encodes a netrin receptor. Cell 87:175-185.

Keleman K, Dickson BJ (2001) Short- and long-range repulsion by the Drosophila Unc5 netrin receptor. Neuron 32:605-617.

Kennedy TE, Serafini T, de la Torre JR, Tessier-Lavigne M (1994) Netrins are diffusible chemotropic factors for commissural axons in the embryonic spinal cord. Cell 78:425-435.

Lefcort F, Bentley D (1987) Pathfinding by pioneer neurons in isolated, opened and mesoderm-free limb buds of embryonic grasshoppers. Dev Biol 119:466-480.

Li W, Lee J, Vikis HG, Lee SH, Liu G, Aurandt J, Shen TL, Fearon ER, Guan JL, Han M, Rao Y, Hong K, Guan KL (2004) Activation of FAK and Src are receptor-proximal events required for netrin signaling. Nat Neurosci 7:1213-1221.

Lin SC, Bergles DE (2004) Synaptic signaling between GABAergic interneurons and oligodendrocyte precursor cells in the hippocampus. Nat Neurosci 7:24-32.

Llambi F, Causeret F, Bloch-Gallego E, Mehlen P (2001) Netrin-1 acts as a survival factor via its receptors UNC5H and DCC. EMBO J 20:2715-2722.
Lu QR, Yuk D, Alberta JA, Zhu Z, Pawlitzky I, Chan J, McMahon AP, Stiles CD, Rowitch DH (2000) Sonic hedgehog-regulated oligodendrocyte lineage genes encoding bHLH proteins in the mammalian central nervous system. Neuron 25:317-329.

Mallon BS, Shick HE, Kidd GJ, Macklin WB (2002) Proteolipid promoter activity distinguishes two populations of NG2-positive cells throughout neonatal cortical development. J Neurosci 22:876-885.

Manitt C, Kennedy TE (2002) Where the rubber meets the road: netrin expression and function in developing and adult nervous systems. Prog Brain Res 137:425-442.

Marin O, Rubenstein JL (2003) Cell migration in the forebrain. Annu Rev Neurosci 26:441-483.

Mazelin L, Bernet A, Bonod-Bidaud C, Pays L, Arnaud S, Gespach C, Bredesen DE, Scoazec JY, Mehlen P (2004) Netrin-1 controls colorectal tumorigenesis by regulating apoptosis. Nature 431:80-84.

Mehlen P, Rabizadeh S, Snipas SJ, Assa-Munt N, Salvesen GS, Bredesen DE (1998) The DCC gene product induces apoptosis by a mechanism requiring receptor proteolysis. Nature 395:801-804.

Mekki-Dauriac S, Agius E, Kan P, Cochard P (2002) Bone morphogenetic proteins negatively control oligodendrocyte precursor specification in the chick spinal cord. Development 129:5117-5130.

Meyer D, Yamaai T, Garratt A, Riethmacher-Sonnenberg E, Kane D, Theill LE, Birchmeier C (1997) Isoform-specific expression and function of neuregulin. Development 124:3575-3586.

Miller RH (1996) Oligodendrocyte origins. Trends Neurosci 19:92-96.

Miller RH (2002) Regulation of oligodendrocyte development in the vertebrate CNS. Prog Neurobiol 67:451-467.

Ohsawa R, Ohtsuka T, Kageyama R (2005) Mash1 and Math3 are required for development of branchiomotor neurons and maintenance of neural progenitors. J Neurosci 25:5857-5865.

Prestoz L, Chatzopoulou E, Lemkine G, Spassky N, Lebras B, Kagawa T, Ikenaka K, Zalc B, Thomas J-L (2004) Control of axonophilic migration of oligodendrocyte precursor cells by Eph-ephrin interaction. Neuron Glia Biol 1:73.

Pringle NP, Richardson WD (1993) A singularity of PDGF alpha-receptor expression in the dorsoventral axis of the neural tube may define the origin of the oligodendrocyte lineage. Development 117:525-533.

Pringle NP, Yu WP, Guthrie S, Roelink H, Lumsden A, Peterson AC, Richardson WD (1996) Determination of neuroepithelial cell fate: induction of the oligodendrocyte lineage by ventral midline cells and sonic hedgehog. Dev Biol 177:30-42.

Qi Y, Cai J, Wu Y, Wu R, Lee J, Fu H, Rao M, Sussel L, Rubenstein J, Qiu M (2001) Control of oligodendrocyte differentiation by the Nkx2.2 homeodomain transcription factor. Development 128:2723-2733.

Ren XR, Ming GL, Xie Y, Hong Y, Sun DM, Zhao ZQ, Feng Z, Wang Q, Shim S, Chen ZF, Song HJ, Mei L, Xiong WC (2004) Focal adhesion kinase in netrin-1 signaling. Nat Neurosci 7:1204-1212.

Rowitch DH (2004) Glial specification in the vertebrate neural tube. Nat Rev Neurosci 5:409-419.

Saueressig H, Burrill J, Goulding M (1999) Engrailed-1 and netrin-1 regulate axon pathfinding by association interneurons that project to motor neurons. Development 126:4201-4212.

Serafini T, Kennedy TE, Galko MJ, Mirzayan C, Jessell TM, Tessier-Lavigne M (1994) The netrins define a family of axon outgrowth-promoting proteins homologous to C. elegans UNC-6. Cell 78:409-424.

Serafini T, Colamarino SA, Leonardo ED, Wang H, Beddington R, Skarnes WC, Tessier-Lavigne M (1996) Netrin-1 is required for commissural axon guidance in the developing vertebrate nervous system. Cell 87:1001-1014.

Shekarabi M, Kennedy TE (2002) The netrin-1 receptor DCC promotes filopodia formation and cell spreading by activating Cdc42 and Rac1. Mol Cell Neurosci 19:1-17.

Small RK, Riddle P, Noble M (1987) Evidence for migration of oligodendrocyte-type-2 astrocyte progenitor cells into the developing rat optic nerve. Nature 328:155-157.

Spassky N, de Castro F, Le Bras B, Heydon K, Queraud-LeSaux F, BlochGallego E, Chedotal A, Zalc B, Thomas JL (2002) Directional guidance of oligodendroglial migration by class 3 semaphorins and netrin-1. J Neurosci 22:5992-6004.

Srinivasan K, Strickland P, Valdes A, Shin GC, Hinck L (2003) Netrin-1/ 
neogenin interaction stabilizes multipotent progenitor cap cells during mammary gland morphogenesis. Dev Cell 4:371-382.

Starz-Gaiano M, Lehmann R (2001) Moving towards the next generation. Mech Dev 105:5-18.

Sugimoto Y, Taniguchi M, Yagi T, Akagi Y, Nojyo Y, Tamamaki N (2001) Guidance of glial precursor cell migration by secreted cues in the developing optic nerve. Development 128:3321-3330.

Tsai HH, Frost E, To V, Robinson S, Ffrench-Constant C, Geertman R, Ransohoff RM, Miller RH (2002) The chemokine receptor CXCR2 controls positioning of oligodendrocyte precursors in developing spinal cord by arresting their migration. Cell 110:373-383.

Tsai HH, Tessier-Lavigne M, Miller RH (2003) Netrin 1 mediates spinal cord oligodendrocyte precursor dispersal. Development 130:2095-2105.

Vallstedt A, Klos JM, Ericson J (2005) Multiple dorsoventral origins of oli- godendrocyte generation in the spinal cord and hindbrain. Neuron 45:55-67.

van Heyningen P, Calver AR, Richardson WD (2001) Control of progenitor cell number by mitogen supply and demand. Curr Biol 11:232-241.

Vartanian T, Fischbach G, Miller R (1999) Failure of spinal cord oligodendrocyte development in mice lacking neuregulin. Proc Natl Acad Sci USA 96:731-735.

Wichterle H, Garcia-Verdugo JM, Alvarez-Buylla A (1997) Direct evidence for homotypic, glia-independent neuronal migration. Neuron 18:779-791.

Zhang H, Miller RH (1996) Density-dependent feedback inhibition of oligodendrocyte precursor expansion. J Neurosci 16:6886-6895.

Zhou Q, Wang S, Anderson DJ (2000) Identification of a novel family of oligodendrocyte lineage-specific basic helix-loop-helix transcription factors. Neuron 25:331-343. 